\title{
The improved quality of postoperative analgesia after intrathecal morphine does not result in improved recovery and quality of life in the first 6 months after orthopedic surgery: a randomized controlled pilot study
}

This article was published in the following Dove Press journal: Journal of Pain Research

9 May 2017

Number of times this article has been viewed

\section{Nilufar Foadi ${ }^{1}, *$ \\ Matthias Karst ${ }^{1, *}$ \\ Anika Frese-Gaul ${ }^{2}$ \\ Niels Rahe-Meyer ${ }^{3}$ \\ Stefan Krömer' \\ Christian Weilbach ${ }^{4}$ \\ 'Department of Anesthesiology and Intensive Care Medicine, Pain Clinic, Hannover Medical School, Hannover, ${ }^{2}$ Department of Psychosomatic Medicine, AHG Psychosomatische Klinik Bad Pyrmont, Bad Pyrmont, ${ }^{3}$ Department of Anesthesiology and Operative Intensive Care Medicine, Franziskus Hospital, Bielefeld, ${ }^{4}$ Department of Anesthesiology, Intensive Care Medicine, Emergency Medicine and Pain Medicine, St. Josefs-Hospital Cloppenburg, Cloppenburg, Germany}

*These authors contributed equally to this work
Correspondence: Nilufar Foadi Department of Anesthesiology and Intensive Care Medicine, Pain Clinic, Hannover Medical School, Carl-Neuberg Strasse I, 30625 Hannover, Germany Tel +49 5 II 5322489 Fax +49 5II 5323642

Email foadi.nilufar@mh-hannover.de
Objective: In orthopedic surgery, it is well known that the use of intrathecal morphine (ITM) leads to an improved quality of postoperative analgesia. Little is known how this improved analgesia affects the long-term course after surgery.

Study design: A randomized, double-blind trial.

Setting: Academic medical center.

Subjects: Forty-nine patients undergoing total hip or knee replacement surgery in spinal anesthesia.

Methods: Patients were randomly assigned to receive either $0.1 \mathrm{mg}(\mathrm{n}=16)$ or $0.2 \mathrm{mg}(\mathrm{n}=16)$ morphine sulfate intrathecally or physiological saline $(\mathrm{n}=17)$ added to $3 \mathrm{~mL} 0.5 \%$ isobaric bupivacaine for spinal anesthesia. As a function of the quality of the short-term postoperative analgesia, the effect on recovery and quality of life was evaluated at various time points up to 26 weeks after surgery.

Results: In both ITM groups, the additionally required postoperative systemic morphine dose was significantly reduced compared with the placebo group $(P=0.004)$. One week after operation, patients with ITM reported significantly less pain at rest $(P=0.01)$ compared to the placebo group. At discharge, in comparison with the $0.1 \mathrm{mg}$ ITM and placebo group, the $0.2 \mathrm{mg}$ ITM group showed a higher degree of impairment regarding pain, stiffness, and physical function of the respective joint $(P=0.02)$. Over the further follow-up period of 6 months after surgery, recovery and the quality of life did not differ significantly between the three study groups $(P>0.2)$. Conclusion: Morphine $(0.1 \mathrm{mg})$ as adjunct to $0.5 \%$ bupivacaine for spinal anesthesia is effective to produce a pronounced postoperative analgesia with a beneficial analgesic effect up to 1 week after surgery. With this study design, the different quality of postoperative analgesia had no effect on quality of life and recovery in patients over the 6-month follow-up period. In the medium term, ITM may induce hyperalgesic effects.

Keywords: intrathecal opioid, adjunct, perioperative analgesia, long-term outcome, recovery

\section{Introduction}

Neuraxial morphine is extensively used in the perioperative setting due to improved analgesia, greater duration of action, and dose-sparing effects when compared with its administration via the systemic route. ${ }^{1}$ Intrathecal morphine (ITM) appears to have an analgesic efficacy "ceiling" with the optimal intrathecal dose of $0.075-0.3 \mathrm{mg} .{ }^{1,2}$ 
Utilizing larger doses may increase adverse effects, especially the risk of respiratory depression, without necessarily improving analgesia. ${ }^{1-3}$

While the prolonged analgesia induced by ITM up to 48 hours after surgery is well documented in the context of various operations, little is known as to whether improved analgesia leads to long-lasting effects on recovery and quality of life in excess of the short-term postoperative setting.

In a previous multicenter study, in which our center contributed a substantial number of eligible patients, Gehling et al investigated the duration of analgesia after ITM in a dose of 0.1 and $0.2 \mathrm{mg}$, as an adjuvant of bupivacaine compared with placebo. ${ }^{4}$ They found that patients who had received either of the ITM doses showed a significant reduction in additional systemic opioid requests compared with placebo during the first three postoperative days. ${ }^{4}$ The analgesic effect was prolonged in the group with higher ITM dose. ${ }^{4}$

The patients enrolled in the current trial were part of the cohort of the multicenter study by Gehling et al. ${ }^{4}$ The primary aim in the study of this cohort was to determine whether the intensified short-term analgesia with ITM induces longterm effects on recovery and quality of life up to 6 months after the operation. The secondary goals were to determine postoperative pain levels at rest and during movement, use of concomitant pain medication, and side effects of ITM.

\section{Methods}

The study was conducted with the approval of the Ethics Committee of the Medical Association of Lower Saxony. Procedures followed the ethical standards of the Helsinki Declaration of the World Medical Association. All patients were informed, both verbally and in writing, about the aim and procedures of the study and gave their written consent to participate in this clinical trial.
Patients of the orthopedic department of the Hannover Medical School (Annastift Hannover) scheduled to undergo total knee or hip replacement surgery for osteoarthritis of the knee or hip were included in this study. The inclusion and exclusion criteria for the three patient groups are listed in Table 1.

The study was designed with three patient groups. For randomization, a multiplicative congruential randomnumber generator and the Visual Basic Rnd function were used. After defining the length and sequence of the block sizes, random numbers generated with the Rnd function of Visual Basic 3.0 were used for the allocation of medication within the blocks.

In addition to the $0.5 \%$ bupivacaine solution for spinal anesthesia, the patients in group I received a placebo solution (saline), group II received $0.2 \mathrm{mg}$ morphine sulfate, and group III received $0.1 \mathrm{mg}$ morphine sulfate (Table 2). Besides patient-reported pain after surgery, reduced requirement for additional pain medication was another criterion used to evaluate the effect of ITM.

Each patient received a dose of $1 \mathrm{~g}$ metamizole (orally or intravenously) every 4 hours as the basal medication for postoperative pain relief. When the basal medication did not provide adequate relief, patients could request a dose of $5 \mathrm{mg}$ morphine (intravenously or subcutaneously) as a rescue medication until the pain was perceived as "well tolerable."

Table 2 Patient groups and study medication

\begin{tabular}{lll}
\hline Group & $\begin{array}{l}\text { Study medication } \\
\text { added to bupivacaine }\end{array}$ & Patients (n) \\
\hline I & Placebo (physiological saline) & 17 \\
II & $0.2 \mathrm{mg}$ morphine sulfate & 16 \\
III & $0.1 \mathrm{mg}$ morphine sulfate & 16 \\
\hline
\end{tabular}

Note: Forty-nine patients were randomly assigned to receive either $0.1 \mathrm{mg}$ (group III) or $0.2 \mathrm{mg}$ (group II) morphine sulfate or physiological saline (group I) intrathecally added to $3 \mathrm{~mL} 0.5 \%$ isobaric bupivacaine for spinal anesthesia.

Table I Inclusion and exclusion criteria of the clinical trial

\begin{tabular}{ll}
\hline Inclusion criteria & Exclusion criteria \\
\hline Age: from I8 years & Systemic infection or local infection at puncture site (SPA) \\
ASA risk group: I-3 & Spinal metastasis \\
Surgery-related hospitalization & Coagulopathy \\
Surgical procedure: total hip or knee replacement surgery in spinal & Daily alcohol consumption $>60 \mathrm{~g}$ (approximately one $500 \mathrm{~mL}$ bottle of \\
anesthesia & wine) \\
Voluntary participation: written informed consent for participation in & Chronic opioid intake \\
the study and data privacy statement & Pregnant or breastfeeding women \\
Willingness and ability to fulfill the requirements of the study protocol & Hypovolemia \\
& Known hypersensitivity to morphine and/or metamizole \\
& Patient refusal before, during, or after surgery \\
& Sleep apnea syndrome \\
\hline
\end{tabular}

Abbreviations: ASA, American Society of Anesthesiologists (risk classification); SPA spinal anesthesia. 


\section{Assessment tools}

The German version of the following standardized selfassessment questionnaires was used to measure quality of life and chronification of pain: the Western Ontario and McMaster Universities Osteoarthritis Index (WOMAC) questionnaire, Numeric Rating Scale (NRS)-series, ${ }^{5}$ the 36-Item Short Form Health Survey (SF-36), ${ }^{6}$ the Beck Depression Inventory (BDI), ${ }^{7}$ and the Clinical Global Impressions (CGI) scale. ${ }^{8}$

These questionnaires, relating either to disease-specific (eg, WOMAC) or unspecific factors (eg, SF-36 and BDI), are listed in Table 3.

\section{Clinical trial process}

The patients were assessed at defined time points (Table 4). On the day before surgery, the patients were informed about the type and technique of anesthesia and risks related to it. The patients then received information about the purpose and process of the planned study and gave their written consent to participate in the trial. Once consent was obtained, the medical history was taken and the following basic data were obtained: age (years), weight $(\mathrm{kg})$, height $(\mathrm{cm})$, gender, whether pregnant/breastfeeding, ethnicity (White/African/ Asian/other), smoking status, concomitant disease and concomitant treatment, and current pain medication.

One day before surgery, the patients received the questionnaires as detailed in Table 4 (pain questionnaire [NRS and tolerability of pain], WOMAC, SF-36 Health Survey, CGI, and $\mathrm{BDI}$ ); instructions were provided on how to complete the questionnaire, where required. Furthermore, location and onset of pain (NRS 0-10) and severity of osteoarthrosis as well as anxiety (from $0=$ no anxiety to $10=$ severe anxiety) were determined.

\section{Short-term evaluation}

Immediately before the induction of spinal anesthesia, the following data were collected: pain intensity (NRS) at present/during the last 24 hours, degree of sedation, nausea/ vomiting, itching, difficulty urinating, and the following vital signs (blood pressure, heart rate, respiratory rate, and peripheral oxygen saturation).

After surgery, the following parameters were recorded at $4,5,6,7,8,12$, and 16 hours after the administration of spinal anesthesia and in the morning of the first, second, and third

Table 3 Descriptions of the self-assessment questionnaires used

\begin{tabular}{ll}
\hline Questionnaire (abbreviation) & Description \\
\hline $\begin{array}{l}\text { Western Ontario and McMaster } \\
\begin{array}{l}\text { Universities Osteoarthritis Index } \\
\text { (WOMAC) questionnaire }\end{array}\end{array}$ & $\begin{array}{l}\text { Multidimensional, disease-specific questionnaire covering the symptoms of patients with osteoarthritis of } \\
\text { the knee and hip; questions on pain intensity, stiffness, and functional impairments. } \\
\text { 36-Item Short Form Health Survey } \\
\text { (SF-36) }\end{array}$ \\
$\begin{array}{ll}\text { Questionnaire for assessment of the health-related quality of life of patients, independent of present } \\
\text { health status and age of the respondent, covering the patient's physical function and well-being. It consists } \\
\text { of eight subscales. }\end{array}$ \\
$\begin{array}{l}\text { Self-assessment instrument designed to measure the severity of depression. } \\
\text { Questionnaire designed to measure treatment success. It includes a seven-point scale that requires the }\end{array}$ \\
\hline
\end{tabular}

Table 4 Timeline of the clinical trial process

\begin{tabular}{|c|c|c|c|c|c|c|c|}
\hline & $\begin{array}{l}\text { Surgery + study } \\
\text { medication } \\
\text { administration }\end{array}$ & & & & & & $\begin{array}{l}\text { End of } \\
\text { study }\end{array}$ \\
\hline Preoperative & Day of surgery & Days I, 2, and 3 & Week I & Week 2 & Discharge & Week 8 & Month 6 \\
\hline $\begin{array}{l}\text { Discussion and } \\
\text { informed consent } \\
\text { undertaken then } \\
\text { exclusion and inclusion } \\
\text { criteria applied. } \\
\text { Questionnaires } \\
\text { used before surgery: } \\
\text { WOMAC, SF-36, and } \\
\text { BDI }\end{array}$ & $\begin{array}{l}\text { Assessment of pain } \\
\text { intensity at rest and } \\
\text { with movement } \\
\text { and tolerability of } \\
\text { pain at } 4,5,6,7,8 \text {, } \\
12 \text {, and } 16 \text { h after } \\
\text { administration of } \\
\text { spinal anesthesia }\end{array}$ & $\begin{array}{l}\text { Assessment of pain } \\
\text { intensity at rest and with } \\
\text { movement and tolerability } \\
\text { of pain }\end{array}$ & $\begin{array}{l}\text { Assessment } \\
\text { of pain } \\
\text { intensity } \\
\text { at rest } \\
\text { and with } \\
\text { movement } \\
\text { and } \\
\text { tolerability } \\
\text { of pain }\end{array}$ & $\begin{array}{l}\text { Assessment } \\
\text { of pain } \\
\text { intensity at } \\
\text { rest and with } \\
\text { movement and } \\
\text { tolerability of } \\
\text { pain }\end{array}$ & $\begin{array}{l}\text { Assessment } \\
\text { of pain } \\
\text { intensity at } \\
\text { rest and with } \\
\text { movement and } \\
\text { tolerability of } \\
\text { pain. WOMAC } \\
\text { and CGI used }\end{array}$ & $\begin{array}{l}\text { WOMAC, } \\
\text { SF-36, } \\
\text { BDI, and } \\
\text { CGI }\end{array}$ & $\begin{array}{l}\text { WOMAC, } \\
\text { SF-36, BDI, } \\
\text { and CGI }\end{array}$ \\
\hline
\end{tabular}

Abbreviations: BDI, Beck Depression Inventory; CGI, Clinical Global Impressions; SF-36, 36-Item Short Form Health Survey; WOMAC, Western Ontario and McMaster Universities Osteoarthritis Index. 
postoperative day: pain intensity at rest and with movement, pain tolerability according to the Functional Pain Scale $(0=$ no pain; $1=$ tolerable, does not prevent any activities; $2=$ tolerable, does prevent some activities; $3=$ intolerable, but can use telephone, watch television, or read; $4=$ intolerable, cannot use telephone, watch television, or read; $5=$ intolerable and unable to verbally communicate because of pain), ${ }^{9}$ grade of sedation, nausea, itching (0-10 NRS score of itching, from $0=$ no itching to $10=$ most severe itching), vital signs (blood pressure, heart rate, respiratory rate, and peripheral oxygen saturation), use of analgesics, intake of other medications, adverse events, and other symptoms.

A decrease in respiratory frequency $<10 /$ minute or a decrease of peripheral oxygen saturation $<90 \%$ was interpreted as respiratory depression. ${ }^{10}$

Arterial hypotension was defined as a reduction of the mean arterial pressure by $>25 \%$ of the baseline value that was obtained immediately before the administration of spinal anesthesia.

\section{Long-term evaluation}

At the end of the first and second week after surgery, pain intensity at rest and with movement, pain tolerability, and other side effects of the treatment were collected from the patients once again. On the day of discharge, the CGI and WOMAC questionnaires were also used.

Eight weeks, and 6 months after surgery, the questionnaires (WOMAC, SF-36 Health Survey, BDI, and CGI), which the patients had completed on the day before the surgery, were sent out to them again (Table 4).

\section{Statistics}

All data are presented as mean \pm standard deviation. Quantitative variables were compared by the Kruskal-Wallis test followed by the Tukey and Kramer post hoc test and qualitative variables were analyzed by the chi-square test. $P$-values $<0.05$ were considered statistically significant.

\section{Results \\ Patient population}

From the total of 54 eligible patients, five patients dropped out subsequently: one because of an additional need of general anesthesia due to a deficient extent of sensory block, two for not receiving ITM because of technical problems during the induction of spinal anesthesia, one due to a preexisting opioid therapy that became apparent subsequently, and one because reported an adverse reaction related to morphine after the surgery.

Data from 49 patients were analyzed in this study; 16 patients were allocated to the study groups I and II and 17 patients to the study group III (Table 2). There were no significant differences between the groups with regard to biometric data (gender, age, size, and body weight), joint treated, use of analgesics, preexisting pain and pain tolerability, anxiety, and parameters of function prior to surgery (Table 5).

Table 5 Evaluation of group differences regarding biometric and pain-related parameters

\begin{tabular}{|c|c|c|c|c|}
\hline Parameter & Group I & Group II & Group III & $P$-value \\
\hline Age (years) & $67.63 \pm 2.45$ & $67.33 \pm 2.87$ & $63.71 \pm 3.14$ & 0.45 \\
\hline Gender & & & & 0.57 \\
\hline Male & 7 & 4 & 6 & \\
\hline Female & 9 & 12 & 11 & \\
\hline Height (cm) & $165.06 \pm 2.21$ & $164.69 \pm 1.98$ & $166.41 \pm 2.94$ & 0.60 \\
\hline Body weight (kg) & $76.88 \pm 2.36$ & $81.50 \pm 4.80$ & $77.35 \pm 3.04$ & 0.87 \\
\hline Treated joint & & & & 0.76 \\
\hline Treated joint: hip & 7 & 9 & 8 & \\
\hline Treated joint: knee & 9 & 7 & 9 & \\
\hline Number of patients using analgesics & 5 & 2 & 2 & 0.46 \\
\hline Walking distance $(\mathrm{m})$ & $4.08 \pm 1.68$ & $3.33 \pm 1.50$ & $3.21 \pm 2.05$ & 0.46 \\
\hline \multicolumn{5}{|c|}{ Pain and anxiety scores assessed at the day before surgery (NRS 0-10): } \\
\hline Pain at rest & $4.5 \pm 2.70$ & $2.62 \pm 2.83$ & $4.12 \pm 3.84$ & 0.32 \\
\hline Pain with movement & $6.46 \pm 2.72$ & $6.44 \pm 2.73$ & $7.53 \pm 2.10$ & 0.43 \\
\hline Pain tolerability & $3.25 \pm 0.58$ & $3.06 \pm 0.85$ & $3.29 \pm 0.59$ & 0.77 \\
\hline Anxiety & $3.50 \pm 3.03$ & $3.44 \pm 3.25$ & $4.87 \pm 3.44$ & 0.38 \\
\hline
\end{tabular}

Notes: Statistical comparison by the chi-square test did not show significant differences in terms of the depicted parameters comparing the three study groups. Group I, placebo, received local anesthetic and intrathecal saline solution instead of morphine; Group II, received local anesthetic and $0.2 \mathrm{mg}$ intrathecal morphine; Group III, received local anesthetic and $0.1 \mathrm{mg}$ intrathecal morphine. Data presented as mean \pm SD or number of patients.

Abbreviation: NRS, Numeric Rating Scale. 


\section{Postoperative pain at rest}

As a trend, patients in the placebo group experienced stronger pain at rest than patients in the active groups in the first 24 hours after surgery.

The highest mean NRS score at rest (3.38 \pm 3.01$)$ within group II ( $0.2 \mathrm{mg}$ ITM) was reached 24 hours after surgery, the highest mean pain level within group III $(0.1 \mathrm{mg}$ ITM) occurred 8 hours after spinal anesthesia (NRS 3.25 \pm 3.94 ), and the highest mean pain score among the patients of the placebo group was observed 7 hours after spinal anesthesia (NRS 4.75 \pm 3.70 ).

Three days after surgery, no statistical differences between the three study groups were noticeable anymore. However, there was a trend toward higher pain levels in the placebo group compared with the active groups.

One week after surgery, the reported pain level at rest was significantly lower in the active groups compared to the placebo group $(P=0.01$; Kruskal-Wallis test: $\mathrm{H}(2, \mathrm{n}=47)$ $=9.24$, Figure 1). At that time point, pain scores at rest were NRS $3.56 \pm 0.76$ for the placebo group, NRS $1.34 \pm 1.95$ for group II ( $0.2 \mathrm{mg}$ ITM), and NRS $1.5 \pm 2.80$ for group III ( 0.1 mg ITM; $P=0.01$; Kruskal-Wallis test: $\mathrm{H}(2, \mathrm{n}=45)=7.99$, Figure 1A).

\section{Postoperative pain during movement}

As expected, pain levels during movement decreased with increasing time interval after surgery in all three study groups (Figure 1B).

Up to the first week after surgery, as a trend, patients in the active groups showed lower levels of pain with movement compared to the patients in the placebo group, but without statistical significance $(P=1.45$, Figure 1B).

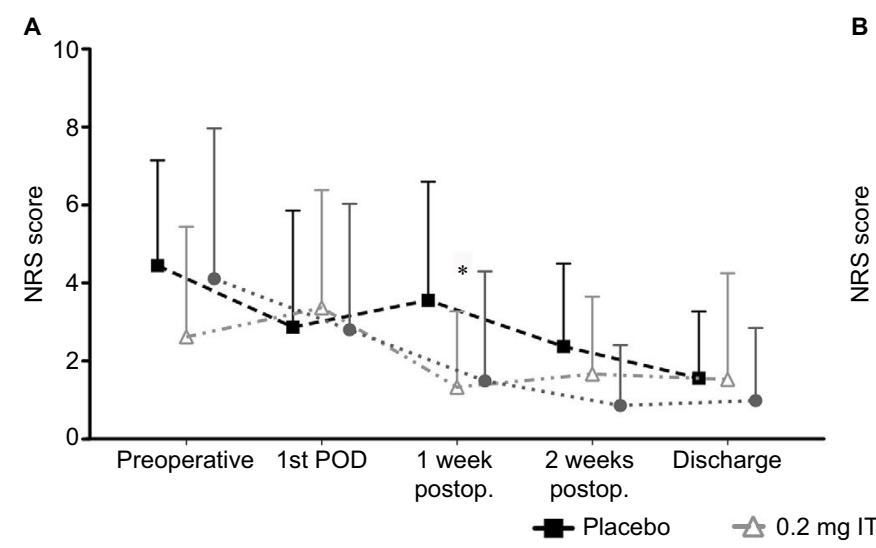

Figure I Pain intensity at the first postoperative day and in the weeks following surgery.

Notes: Mean values of pain intensity at rest $(\mathbf{A})$ and during movement $(\mathbf{B})$ in the short-term postoperative course are displayed. Error bars depict SD. Pain scores were assessed I day before surgery (preoperative), $24 \mathrm{~h}$ after spinal anesthesia (Ist POD), I and 2 weeks after surgery, and at the day of discharge. The mean value for hospital stay was $18.68 \pm 1.63$ days $(n=47)$ without significant difference comparing the three study groups. Significantly lower pain values in the active groups compared with the placebo group are marked; $* P<0.02$.

Abbreviations: SD, standard deviation; POD, postoperative day, NRS, Numeric Rating Scale, postop., postoperative, ITM, intrathecal morphine.

\section{Tolerability of pain}

At 4, 5, 6, 7, and 8 hours after spinal anesthesia, patients who had received $0.2 \mathrm{mg}$ ITM rated their pain as significantly more tolerable than those with additional placebo (Figure 2A; $P<0.01$; Kruskal-Wallis test: $\mathrm{H}(2, \mathrm{n}=49)=12.97$ for 4 hours, 16.65 for 5 hours; 18.55 for 6 hours; 16.5 for 7 hours; 8.12 for 8 hours). There was no statistical difference between the placebo group and the patients who had received $0.1 \mathrm{mg}$ ITM $(P>0.07$; Figure 2A).

At 12-16 hours after spinal anesthesia and also on the first, second, and third postoperative day, as well as 1 and 2 weeks after surgery and at the day of discharge, there were no significant differences regarding pain tolerability between the three different patient groups (Figure 2A).

\section{Postoperative need for additional analgesics}

The additionally required cumulative intravenous morphine dosage was significantly reduced in the active groups compared with the placebo group until the morning of the first postoperative day $(P=0.004$; Kruskal-Wallis test: $\mathrm{H}(2, \mathrm{n}=49)$ $=11.10$, Figure 2B). The systemic morphine consumption did not significantly differ between the two active groups.

In the active groups, $62.5 \%$ and $52.9 \%$ of the patients in the $0.2 \mathrm{mg}$ morphine and $0.1 \mathrm{mg}$ morphine group, respectively, needed no additional intravenous morphine, while only $6.25 \%$ of patients in the placebo group managed without additional intravenous morphine.

Comparing the systemic morphine consumption during the second and third postoperative days, no significant differences between the three study groups were detected any more.

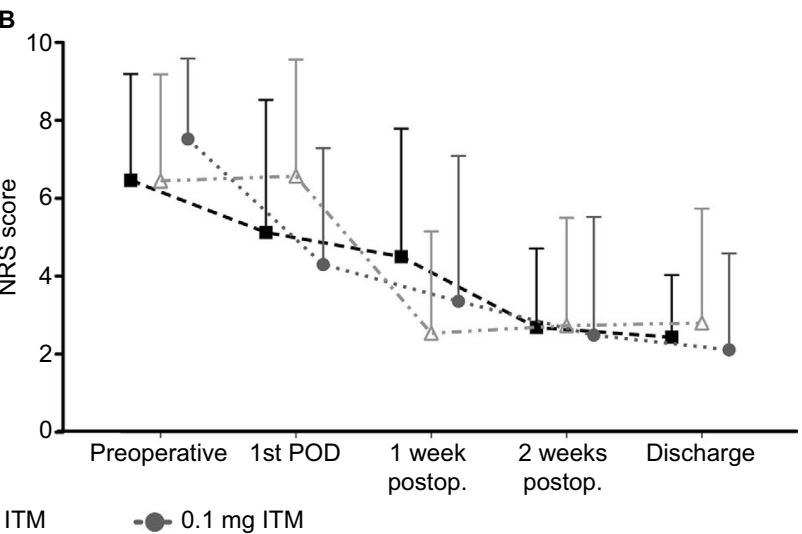



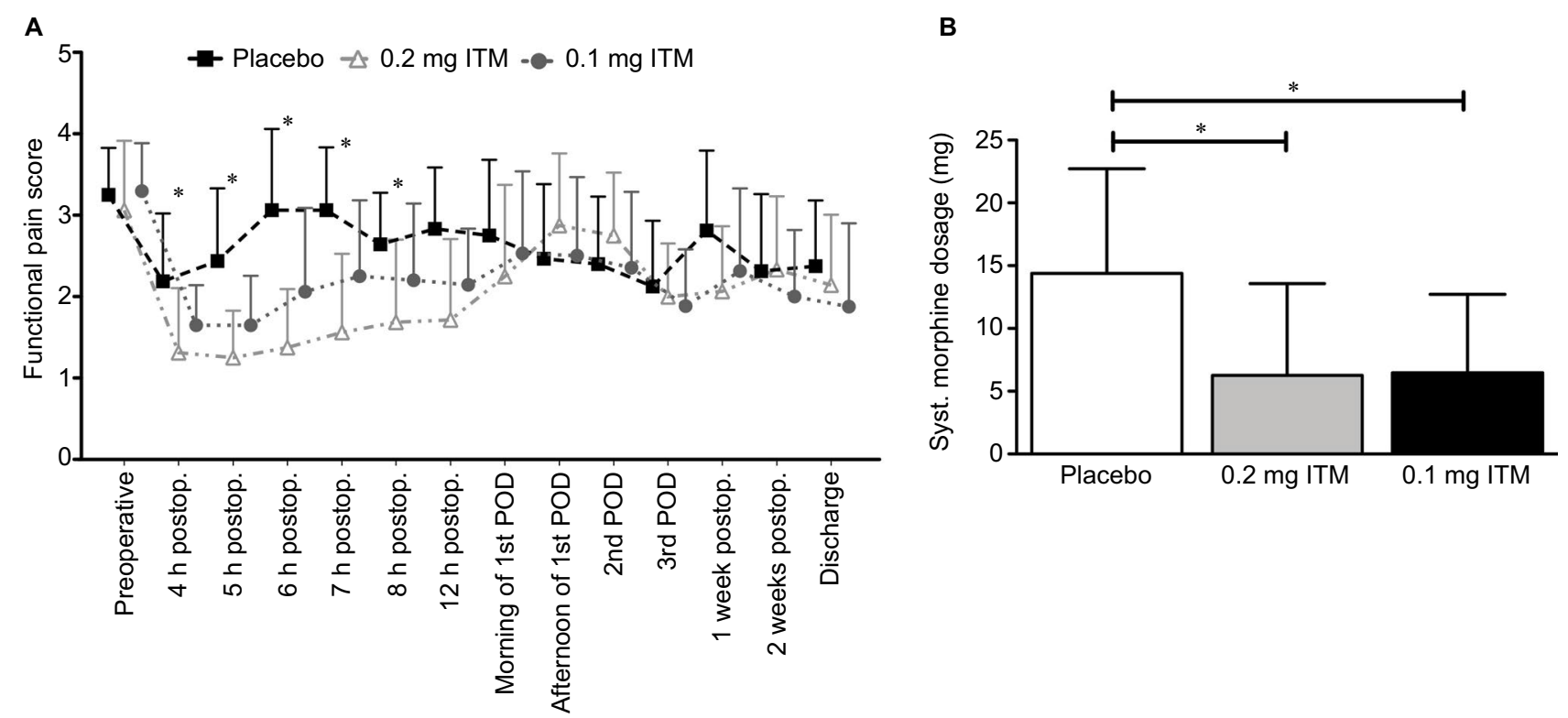

Figure 2 Evaluation of pain tolerability and morphine consumption.

Notes: (A) Pain tolerability was assessed according to the Functional Pain Scale 9 I day before surgery and at the depicted time points. Mean values and SD (error bars) are illustrated. Up to $6 \mathrm{~h}$ after spinal anesthesia, patients of the active groups rated their pain tolerability higher than the patients of the placebo group rated theirs ( $* P<0.0 \mathrm{I}$ ). (B) The additional systemic morphine dose that was cumulatively required within the first $24 \mathrm{~h}$ after surgery was significantly higher in the placebo group compared with the active groups $(* P=0.004)$.

Abbreviations: SD, standard deviation; ITM, intrathecal morphine; postop., postoperative; POD, postoperative day; Syst., systemic.

In the placebo group, four patients received additional pain medication (two patients received $100 \mathrm{mg}$ diclofenac, one patient received $5 \mathrm{mg}$ piritramide, and one patient received $1000 \mathrm{mg}$ paracetamol). In the active groups, only one patient from the $0.1 \mathrm{mg}$ morphine group received a dose of $600 \mathrm{mg}$ ibuprofen.

\section{Side effects of pain medication}

In none of the study groups, clinically relevant episodes of respiratory depression, sedation, and postoperative nausea and vomiting were observed. In general, no major adverse events became apparent.

At 6 and 8 hours after the induction of spinal anesthesia, no itching occurred within the placebo group while five of sixteen patients (31.25\%) in the $0.2 \mathrm{mg}$ morphine group reported itching. For the $0.1 \mathrm{mg}$ morphine group, there were no differences in the occurrence of itching compared with the placebo and the $0.2 \mathrm{mg}$ morphine group. Among all the study groups, no itching was reported from the second postoperative day onward.

\section{Length of stay in hospital}

The duration of stay in hospital did not differ between the three study groups $(P=0.45)$ : the length of stay in hospital was $18.38 \pm 1.20$ days in the placebo group $(n=16), 19.25 \pm 2.05$ days in the group with $0.1 \mathrm{mg}$ ITM $(\mathrm{n}=16)$, and $18.40 \pm 1.45$ days in the group with $0.2 \mathrm{mg}$ ITM ( $\mathrm{n}=15)$.

\section{Questionnaires WOMAC}

Preoperatively, no significant differences were detectable between the three study groups.

In each of the three WOMAC subscales (pain, stiffness, and physical function), improvements were observed in all the study groups at the time of discharge and at week 8 and 6 months after surgery compared with the baseline scores prior to surgery (Figure 3).

In comparison with groups I and III, patients of group II (0.2 mg ITM) showed higher NRS scores regarding pain, stiffness, and functional limitation at discharge (comparison of group II and III: $P=0.02$; Kruskal-Wallis test: $\mathrm{H}(2, \mathrm{n}=46)$ $=7.59$ for pain, 7.48 for stiffness, and 7.40 for physical function) while there was no significant difference comparing groups I and III (Figure 3).

\section{SF-36 Health Survey}

The extensive data collected with this index showed continuous improvement in quality of life after surgery, with the best scores being recorded at 6 months after surgery (Table 6). 

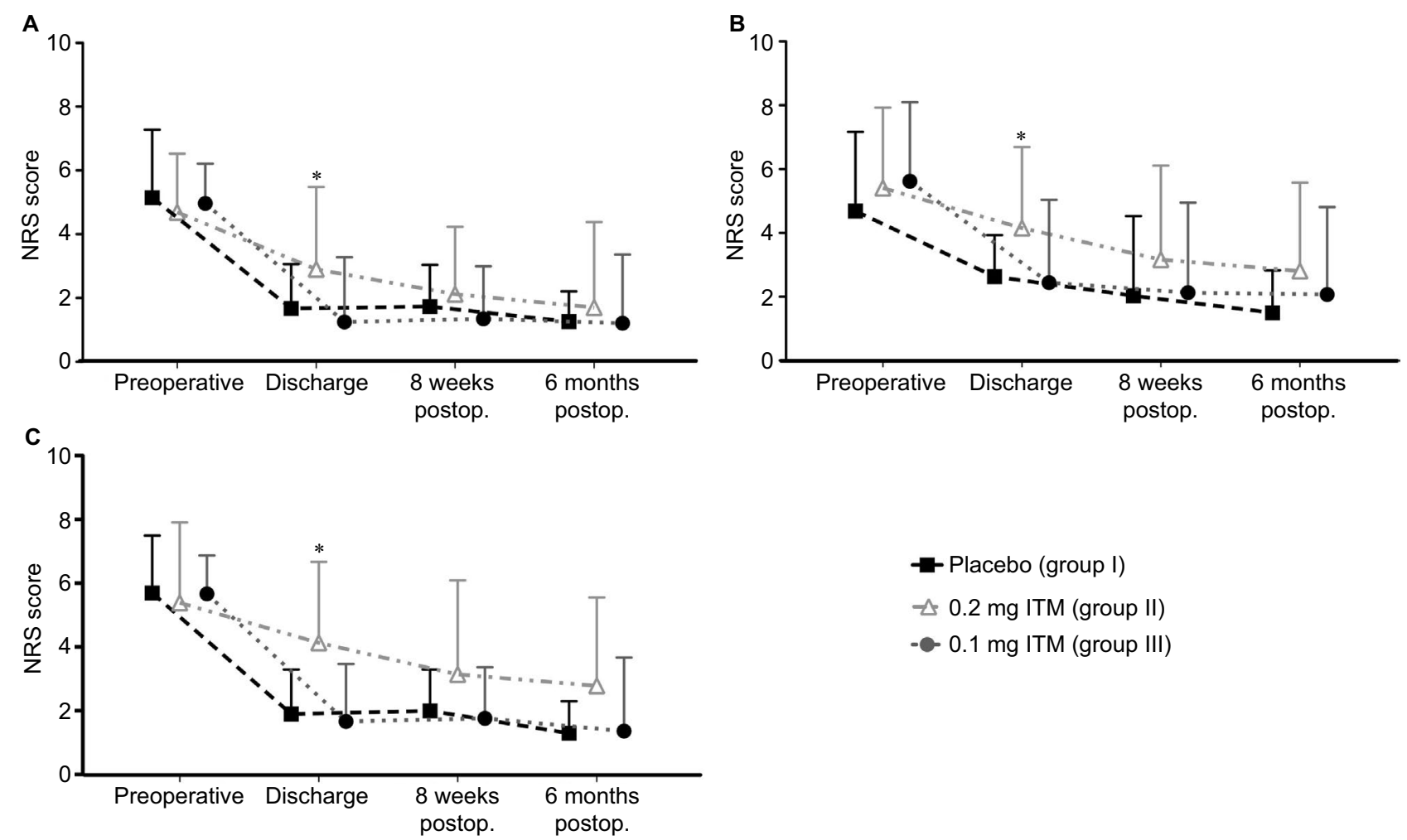

Figure 3 WOMAC questionnaire scores.

Notes: Final mean subscale values (NRS 0-10 with $0=$ no pain/impairment, $10=$ worst pain/impairment possible) derived from the WOMAC questionnaire are depicted for three dimensions: pain (A), stiffness $(\mathbf{B})$, and physical function $(\mathbf{C})$. The respective questionnaires were answered I day before surgery (preoperative), at discharge, 8 weeks, and 6 months after surgery. At discharge the score in group II was significantly higher compared to the score in the other two groups $(P=0.02)$. The mean length of stay in hospital was $18.68 \pm 1.63$ days $(n=47)$.

Abbreviations: WOMAC, Western Ontario and McMaster Universities Osteoarthritis Index; NRS, Numeric Rating Scale; ITM, intrathecal morphine; postop., postoperative.

No significant differences were found between the placebo group and the active groups.

\section{$\mathrm{BDI}$}

Already before surgery, patients of all three study groups had a higher mean depression score $(8.66 \pm 0.70, n=47)$ than that described for healthy control groups (reported mean score 6.5). ${ }^{11}$ At 6 months after surgery, the mean score of groups I and III was reduced to a value found in healthy control groups ${ }^{11}$ while patients in the $0.2 \mathrm{mg}$ morphine group had higher scores pointing to a higher degree of depression (Figure 4A). At 8 weeks after surgery, the score of group II was $9.77 \pm 1.84(\mathrm{n}=13)$ while the score of group III was $5.08 \pm 1.34$ $(\mathrm{n}=13$, Figure 4A; Kruskal-Wallis test: $\mathrm{H}(2, \mathrm{n}=35)=3.63$; $P=0.16$ ); however, the absolute score of 10.2 (group II) was still below the cutoff score (11.0) for mild depression. ${ }^{7}$

\section{CGI}

At discharge and 6 months after surgery, treatment success was not rated differently comparing the three study groups (Figure 4B). Eight weeks after surgery, significantly lower treatment success ratings were assessed for the patients in the $0.2 \mathrm{mg}$ morphine group compared with the patients of the other two study groups $(P=0.03$; Kruskal-Wallis test: $\mathrm{H}$ $(2, n=46)=7.82$, Figure 4B).

\section{Discussion}

In this study, a superior pain relief in the ITM groups was observable as a short-term effect, which was already achieved with a dosage of $0.1 \mathrm{mg}$ ITM. The advanced analgesic effect was evident in the first 12 postoperative hours and 1 week after surgery. These results are consistent with the data of a meta-analysis of 65 trials on opioids added to local anesthetics for different surgical procedures ${ }^{12}$ : ITM $(0.05-2 \mathrm{mg})$ as adjunct to bupivacaine was capable of prolonging the duration of postoperative analgesia up to the 12th postoperative hour and to reduce postoperative systemic morphine consumption. ${ }^{12}$ The postoperative analgesia provided by ITM without local anesthetic during the first 24 postoperative hours has even been considered superior to either administration of intravenous ketamine at low doses or a regimen of postoperative nonsteroidal 
Table 6 SF-36 scores (mean \pm SD)

\begin{tabular}{|c|c|c|c|c|c|c|c|c|c|}
\hline \multirow[t]{2}{*}{ SF-36 dimension } & \multicolumn{3}{|l|}{ Group I } & \multicolumn{3}{|l|}{ Group II } & \multicolumn{3}{|l|}{ Group III } \\
\hline & Preop. & 8 weeks & 6 months & Preop. & 8 weeks & 6 months & Preop. & 8 weeks & 6 months \\
\hline Physical functioning & $\begin{array}{l}23.13 \pm 15.59 \\
n=16\end{array}$ & $\begin{array}{l}47.00 \pm 22.26 \\
n=15\end{array}$ & $\begin{array}{l}53.93 \pm 20.40 \\
n=14\end{array}$ & $\begin{array}{l}25.00 \pm 18.35 \\
n=16\end{array}$ & $\begin{array}{l}33.67 \pm 22.00 \\
n=15\end{array}$ & $\begin{array}{l}46.54 \pm 26.49 \\
n=13\end{array}$ & $\begin{array}{l}21.76 \pm 12.98 \\
n=17\end{array}$ & $\begin{array}{l}47.50 \pm 25.10 \\
n=16\end{array}$ & $\begin{array}{l}56.00 \pm 30.13 \\
n=15\end{array}$ \\
\hline Role physical & $\begin{array}{l}10.94 \pm 25.77 \\
n=16\end{array}$ & $\begin{array}{l}32.69 \pm 44.94 \\
n=13\end{array}$ & $\begin{array}{l}53.57 \pm 40.26 \\
n=14\end{array}$ & $\begin{array}{l}20.31 \pm 35.61 \\
n=16\end{array}$ & $\begin{array}{l}16.67 \pm 32.27 \\
n=15\end{array}$ & $\begin{array}{l}48.08 \pm 38.81 \\
n=13\end{array}$ & $\begin{array}{l}17.65 \pm 31.58 \\
n=17\end{array}$ & $\begin{array}{l}19.23 \pm 32.52 \\
n=13\end{array}$ & $\begin{array}{l}60.42 \pm 47.02 \\
n=12\end{array}$ \\
\hline Role mental & $\begin{array}{l}43.75 \pm 45.08 \\
n=16\end{array}$ & $\begin{array}{l}58.33 \pm 45.23 \\
n=12\end{array}$ & $\begin{array}{l}82.05 \pm 37.55 \\
n=13\end{array}$ & $\begin{array}{l}50.00 \pm 48.69 \\
n=16\end{array}$ & $\begin{array}{l}30.95 \pm 42.29 \\
n=14\end{array}$ & $\begin{array}{l}71.79 \pm 44.82 \\
n=13\end{array}$ & $\begin{array}{l}49.02 \pm 47.31 \\
n=17\end{array}$ & $\begin{array}{l}41.03 \pm 45.45 \\
n=13\end{array}$ & $\begin{array}{l}72.73 \pm 46.71 \\
n=11\end{array}$ \\
\hline Social functioning & $\begin{array}{l}57.81 \pm 33.50 \\
n=16\end{array}$ & $\begin{array}{l}74.17 \pm 25.65 \\
n=15\end{array}$ & $\begin{array}{l}75.89 \pm 15.08 \\
n=14\end{array}$ & $\begin{array}{l}62.50 \pm 31.62 \\
n=16\end{array}$ & $\begin{array}{l}72.50 \pm 23.24 \\
n=15\end{array}$ & $\begin{array}{l}70.19 \pm 30.42 \\
n=13\end{array}$ & $\begin{array}{l}66.91 \pm 31.23 \\
n=17\end{array}$ & $\begin{array}{l}76.56 \pm 26.95 \\
n=16\end{array}$ & $\begin{array}{l}74.17 \pm 29.30 \\
n=15\end{array}$ \\
\hline Mental health & $\begin{array}{l}70.75 \pm 18.23 \\
n=16\end{array}$ & $\begin{array}{l}74.57 \pm 20.49 \\
n=14\end{array}$ & $\begin{array}{l}76.29 \pm 18.33 \\
n=14\end{array}$ & $\begin{array}{l}62.50 \pm 18.29 \\
n=16\end{array}$ & $\begin{array}{l}69.54 \pm 21.76 \\
n=13\end{array}$ & $\begin{array}{l}74.67 \pm 24.62 \\
n=2\end{array}$ & $\begin{array}{l}70.35 \pm 20.35 \\
n=17\end{array}$ & $\begin{array}{l}79.73 \pm 17.79 \\
n=15\end{array}$ & $\begin{array}{l}76.00 \pm 18.02 \\
n=15\end{array}$ \\
\hline Vitality & $\begin{array}{l}50.31 \pm 20.20 \\
n=16\end{array}$ & $\begin{array}{l}62.67 \pm 18.31 \\
n=15\end{array}$ & $\begin{array}{l}66.43 \pm 16.92 \\
n=14\end{array}$ & $\begin{array}{l}49.38 \pm 22.05 \\
n=16\end{array}$ & $\begin{array}{l}52.31 \pm 22.14 \\
n=13\end{array}$ & $\begin{array}{l}52.50 \pm 20.50 \\
n=12\end{array}$ & $\begin{array}{l}50.00 \pm 24.12 \\
n=17\end{array}$ & $\begin{array}{l}62.00 \pm 22.02 \\
n=15\end{array}$ & $\begin{array}{l}66.67 \pm 21.68 \\
n=15\end{array}$ \\
\hline Bodily pain & $\begin{array}{l}23.25 \pm 17.76 \\
n=16\end{array}$ & $\begin{array}{l}59.71 \pm 23.91 \\
n=14\end{array}$ & $\begin{array}{l}67.43 \pm 20.17 \\
n=14\end{array}$ & $\begin{array}{l}28.19 \pm 24.32 \\
n=16\end{array}$ & $\begin{array}{l}42.80 \pm 19.65 \\
n=15\end{array}$ & $\begin{array}{l}57.54 \pm 23.69 \\
n=13\end{array}$ & $\begin{array}{l}22.88 \pm 10.11 \\
n=17\end{array}$ & $\begin{array}{l}58.07 \pm 26.78 \\
n=15\end{array}$ & $\begin{array}{l}66.40 \pm 26.30 \\
n=15\end{array}$ \\
\hline General health & $\begin{array}{l}58.81 \pm 17.33 \\
n=16\end{array}$ & $\begin{array}{l}65.47 \pm 18.08 \\
n=15\end{array}$ & $\begin{array}{l}65.29 \pm 17.02 \\
n=14\end{array}$ & $\begin{array}{l}61.38 \pm 18.45 \\
n=16\end{array}$ & $\begin{array}{l}58.75 \pm 19.18 \\
n=12\end{array}$ & $\begin{array}{l}61.50 \pm 20.27 \\
n=12\end{array}$ & $\begin{array}{l}63.24 \pm 19.79 \\
n=17\end{array}$ & $\begin{array}{l}68.67 \pm 20.45 \\
n=15\end{array}$ & $\begin{array}{l}71.29 \pm 19.02 \\
n=14\end{array}$ \\
\hline Phys. compon. sum. & $\begin{array}{l}35.46 \pm 12.66 \\
n=16\end{array}$ & $\begin{array}{l}54.86 \pm 19.15 \\
n=13\end{array}$ & $\begin{array}{l}60.61 \pm 17.56 \\
n=14\end{array}$ & $\begin{array}{l}38.72 \pm 15.06 \\
n=16\end{array}$ & $\begin{array}{l}40.35 \pm 16.29 \\
n=12\end{array}$ & $\begin{array}{l}52.38 \pm 19.96 \\
n=12\end{array}$ & $\begin{array}{l}37.03 \pm 11.31 \\
n=17\end{array}$ & $\begin{array}{l}52.04 \pm 20.09 \\
n=13\end{array}$ & $\begin{array}{l}65.51 \pm 22.72 \\
n=12\end{array}$ \\
\hline Mental comp. sum. & $\begin{array}{l}60.16 \pm 16.34 \\
n=16\end{array}$ & $\begin{array}{l}69.79 \pm 19.03 \\
n=12\end{array}$ & $\begin{array}{l}74.18 \pm 14.74 \\
n=13\end{array}$ & $\begin{array}{l}57.14 \pm 20.29 \\
n=16\end{array}$ & $\begin{array}{l}61.13 \pm 18.93 \\
n=13\end{array}$ & $\begin{array}{l}66.52 \pm 22.87 \\
n=12\end{array}$ & $\begin{array}{l}61.45 \pm 20.81 \\
n=17\end{array}$ & $\begin{array}{l}69.64 \pm 19.74 \\
n=13\end{array}$ & $\begin{array}{l}76.62 \pm 18.99 \\
n=11\end{array}$ \\
\hline
\end{tabular}

Notes: SF-36 is a short-form health survey with 36 questions. It was employed I day before surgery (preop.) and at 8 weeks and 6 months after surgery. Comparing the SF-score values of the three groups, no significant difference was found ( $P>0.12$; Kruskal-Wallis $\mathrm{H}$ test). Group I, placebo, received local anesthetic and intrathecal saline solution instead of morphine; Group II, received local anesthetic and $0.2 \mathrm{mg}$ intrathecal morphine; Group III, received local anesthetic and $0.1 \mathrm{mg}$ intrathecal morphine.

Abbreviations: Mental comp. sum., mental component summary score; Phys. compon. sum., physical component summary score; Preop., preoperative; SD, standard deviation; SF-36, 36-Item Short Form Health Survey.
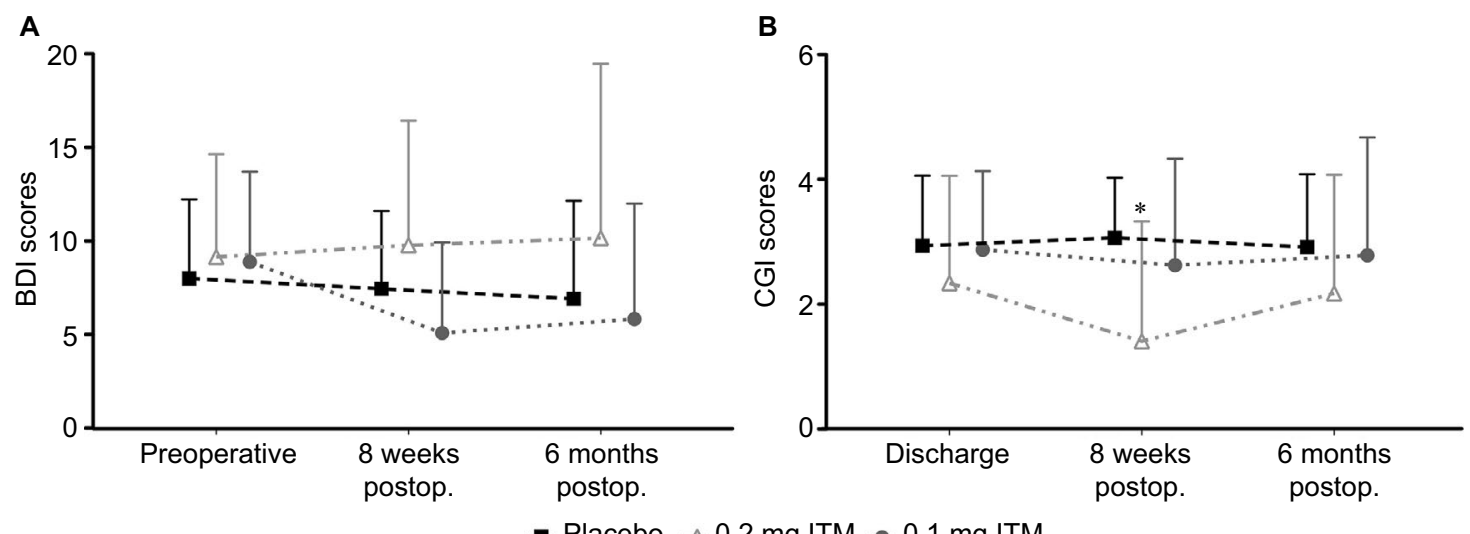

Figure $4 \mathrm{BDI}$ and $\mathrm{CGI}$ scores.

Notes: Mean values and SD (error bars) are presented for BDI (A) and CGI (B) scores. Eight weeks after surgery, treatment success of patients who had received 0.2 mg ITM (group II) was rated lower compared with that of the other two study groups $(* P=0.03)$. From 8 weeks after surgery, patients in the 0.2 mg ITM group showed higher score values for BDI compared to the other two study groups, but without statistically significant difference $(P=0.16)$.

Abbreviations: BDI, Beck Depression Inventory; CGI, Clinical Global Impressions; SD, standard deviation; ITM, intrathecal morphine; postop., postoperative.

anti-inflammatory drugs or a continuous epidural infusion technique. ${ }^{13}$

In this study, the improved perioperative pain control in the ITM groups did not lead to a shorter length of hospital stay. Regarding the length of hospital stay, previous clinical trials showed inconclusive results. ${ }^{14,15} \mathrm{~A}$ meta-analysis by Meylan et al pointed to a slightly decreased duration of hospital stay by 0.5 days in patients with ITM. ${ }^{13}$
Spinal bioavailability is higher for hydrophilic opioids such as morphine enabling not only prolonged segmental analgesia but also rostral spread in spinal cerebrospinal fluid resulting in dose-limiting side effects (eg, sedation and respiratory depression).${ }^{16}$ In this study using ITM below $0.3 \mathrm{mg}$, no relevant respiratory depression was observed. There were also no significant differences in adverse effects requiring supplemental treatment comparing the three study groups. 
However, ITM seems to be associated with an increased risk of respiratory depression, nausea, vomiting, urinary retention, and pruritus. ${ }^{12}$ To reduce the risk of respiratory depression, a ceiling dose of $\leq 0.3 \mathrm{mg}$ was advised. ${ }^{17}$ So far, no significant impact of ITM on perioperative morbidity could be detected. ${ }^{18}$ Perioperative renal, pulmonary, and cardiac complications or mortality in high-risk patients has not been influenced by the use of ITM. ${ }^{19}$ Nevertheless, according to the procedure-specific postoperative pain management recommendations for total knee arthroplasty, ITM is "not recommended as the first choice because of greater potential for adverse events compared with femoral nerve block." ${ }^{20}$

The main result of this study is that an advanced pain relief due to intrathecally administered morphine was not associated with beneficial long-term effects in terms of quality of life or physical function in the further follow-up till 6 months after knee or hip surgery.

It appears that the higher ITM dosage might be disadvantageous since, for the patients of the group with $0.2 \mathrm{mg}$ ITM, poorer WOMAC scores and a lower rating of the treatment success were observable in the follow-up.

The higher postoperative WOMAC score values in the $0.2 \mathrm{mg}$ ITM group assessed at discharge may point to painsensitizing effects of morphine and can lead to the question of whether morphine is a suitable analgesic for intrathecal administration to produce long-term beneficial effects. Although providing a prolonged pain relief in the acute postoperative phase, structural characteristics of the phenanthrene morphine have been shown to favor hyperalgesic effects. ${ }^{21,22}$ The enhancement of pain sensitivity by opioids has been intensively investigated during the last decades. There is evidence that even a single dose of an opioid might cause hyperalgesia. ${ }^{21-24}$ However, experimental studies exploring acute or transient hyperalgesic effects of single doses of morphine on humans are rare. ${ }^{25,26}$

In this study, the cumulative systemic morphine dosage additionally needed by the patients in the placebo group in the first 24 postoperative hours was almost equivalent compared to the dosage of $0.2 \mathrm{mg}$ intrathecally applied morphine (Figure 2B). ${ }^{16}$ However, only a fraction of the intravenously administered morphine reaches spinal receptors and the consecutive response profiles of the intravenously applied morphine and ITM are hardly comparable due to the different time points of application (before and after operation, respectively) and time intervals (one single intrathecal application vs titrated intravenous administration in the course of 24 hours).

So far, to the best of our knowledge, sensory qualities like hyperalgesia and allodynia have not been investigated in a controlled clinical trial in the postoperative course after a single intrathecal low-dose administration of morphine.

While the majority of the studies on ITM evaluated postoperative pain scores and additional systemic opioid consumption for up to 48 hours postoperatively, only limited data are available with regard to long-term effects of ITM. Independent of the surgical procedure and the mode of the control procedures, several studies did not find a prolonged superior analgesic effect of ITM during the first postoperative phase. ${ }^{18,27-31}$ In a prospective observational study by Moriyama et al, no effect on acute pain was observed, but ITM was associated with a decreased prevalence of pain 3 months after a cesarean section. ${ }^{32}$ However, the interpretation of the study results is complicated due to the nonrandomized nature of the study. The intrathecal administration of morphine was an individual choice of the attending anesthesiologist. Furthermore, all patients receiving spinal anesthesia were administered not only $12 \mathrm{mg}$ bupivacaine but additionally $10 \mu \mathrm{g}$ fentanyl intrathecally (with or without $0.1 \mathrm{mg}$ of morphine); besides, postoperatively, different types of supplementary analgesics and different pathways of administration were chosen (pentazocine (30 mg intravenously), flurbiprofen (50 $\mathrm{mg}$ intravenously), loxoprofen (60 mg orally), or acetaminophen (400 mg orally)). ${ }^{32}$

One limitation of this study is the small sample size after the randomization of 49 patients into three study groups; indeed, using the Mann-Whitney $U$ test described by Fan et $\mathrm{al}^{33}$ the post hoc power analysis for comparison of the WOMAC scores of the three study groups 6 months after surgery obtained a power of $17.8 \%$ regarding assessment of the pain subscale, $27.4 \%$ for the dimension of stiffness, and $36.0 \%$ with regard to the physical function score, pointing to an underpowered study. However, the orthopedic hospital at which this study was conducted provided the majority of patients who were enrolled in the multicenter study by Gehling et $\mathrm{al}^{4}$ and was the only one that had appropriate resources of medical staff, enabling the additional assessments. On a positive note, in the follow-up, there were only a few dropouts. The rate of return of the questionnaires stayed stable in the long-term follow-up and was $>70 \%$ up to 6 months after surgery (the different questionnaires were submitted by at least 36 of the 49 patients at different time points). However, to finally assess the long-term effects of ITM, there is a need for studies with a sample size $>100$ revealed by the post hoc power calculation.

There are different possible confounding factors in the perioperative setting and in the postoperative rehabilitation phase identified in several studies investigating the long-term outcome after hip and knee replacement. ${ }^{34-36}$ Factors known 
to be associated with improved short- and medium-term outcomes are a reduced pain level, a better physical function, general health state, emotional and social function, motivation, and self-efficacy. ${ }^{34}$ The postoperative physical rehabilitation was a variable that had not been standardized throughout the three patient groups in this trial. Postoperative recovery can be accelerated by standardized rehabilitative protocols. ${ }^{37}$ Future studies should allow for the difficulty to differentiate, if beneficial postoperative effects can be ascribed to an improved analgesia or to the use of structured inpatient clinical pathways including intensified rehabilitative protocols. ${ }^{37}$

Previous clinical studies including benchmark projects with large sample sizes made evident that postoperative pain differs after total hip and knee replacement surgery. ${ }^{38,39}$ The majority of patients experience moderate to severe pain after total knee replacement, which is associated with a higher risk for developing chronic pain, compared to the patients undergoing total hip replacement. ${ }^{40}$ In the context of this study, there was no unequal distribution among the three study groups in terms of the operated joint (Table 5), but a subgroup analysis was not performed in the follow-up to evaluate possible differences with respect to the operated joint.

Furthermore, somatosensory profiles of the patients were not investigated, which could have revealed possible sensitization processes induced by ITM. Hence, further studies are needed to systematically characterize the long-term effects of ITM with regard to different operative procedures.

\section{Conclusion}

This study showed that the addition of morphine to bupivacaine for spinal anesthesia improved the immediate quality of postoperative analgesia for up to 1 week after surgery. In doing so, the intrathecal application of $0.1 \mathrm{mg}$ morphine was equally effective as the $0.2 \mathrm{mg}$ morphine dose. With this study design and the survey instruments used, no long-lasting improvement in quality of life or physical function resulting from this superior postoperative analgesia was demonstrated in the course of the first 6 months after surgery. On the contrary, it appears that in the medium term higher doses of ITM might promote pain-sensitizing effects.

\section{Acknowledgments}

The authors gratefully acknowledge Dr Wolfgang Reimers for an excellent statistical analysis and also thank the patients whose participation enabled us to conduct this study.

\section{Disclosure}

The authors report no conflicts of interest in this work.

\section{References}

1. Bujedo BM. Spinal opioid bioavailability in postoperative pain. Pain Pract. 2014;14(4):350-364.

2. Sultan P, Gutierrez MC, Carvalho B. Neuraxial morphine and respiratory depression: finding the right balance. Drugs. 2011;71(14):1807-1819.

3. Fares KM, Mohamed SA, Abdel-Ghaffar HS. High dose intrathecal morphine for major abdominal cancer surgery: a prospective doubleblind, dose-finding clinical study. Pain Physician. 2014;17(3):255-264.

4. Gehling MH, Luesebrink T, Kulka PJ, Tryba M. The effective duration of analgesia after intrathecal morphine in patients without additional opioid analgesia: a randomized double-blind multicentre study on orthopedic patients. Eur J Anaesthesiol._2009;26(8):683-688.

5. Bellamy N, Buchanan WW, Goldsmith CH, Campbell J, Stitt L. Validation study of WOMAC: a health status instrument for measuring clinically-import patient-relevant outcomes following total hip or knee arthroplasty in osteoarthritis. J Orthopaedic Rheumatol. 1988;1: 95-108.

6. Bullinger M. German translation and psychometric testing of the SF-36 Health Survey: preliminary results from the IQOLA Project. International Quality of Life Assessment. Soc Sci Med. 1995;41(10): 1359-1366.

7. Beck AT, Ward CH, Mendelson M, Mock J, Erbaugh J. An inventory for measuring depression. Arch Gen Psychiatry. 1961;4:561-571.

8. Guy W, editor. Clinical Global impressions. In: ECDEU Assessment Manual for Psychopharmacology; Revised, 1976. Rockville, MD: US Department of Health Education and Welfare; Public Health Service; Alcohol, Drug Abuse, and Mental Health Administration, National Institute of Mental Health; Psychopharmacology Research Branch, Division of Extramural Research Program; 1976:218-222.

9. Gloth FM 3rd, Scheve AA, Stober CV, Chow S, Prosser J. The Functional Pain Scale: reliability, validity, and responsiveness in an elderly population. J Am Med Dir Assoc. 2001;2(3):110-114.

10. Abouleish E, Rawal N, Tobon-Randall B, et al. A clinical and laboratory study to compare the addition of $0.2 \mathrm{mg}$ of morphine, $0.2 \mathrm{mg}$ of epinephrine, or their combination to hyperbaric bupivacaine for spinal anesthesia in cesarean section. Anesth Analg. 1993;77(3): $457-462$.

11. Hautzinger M, Bailer M, Worall H, Keller F. Beck-Depressions-Inventar BDI. Testhandbuch [Beck's inventory for measuring depression. Manual of tests]. 2nd ed. Bern: Huber; 1995. German.

12. Pöpping DM, Elia N, Marret E, Wenk M, Tramèr MR. Opioids added to local anesthetics for single-shot intrathecal anesthesia in patients undergoing minor surgery: a meta-analysis of randomized trials. Pain. 2012; 153(4):784-793.

13. Meylan N, Elia N, Lysakowski C, Tramèr MR. Benefit and risk of intrathecal morphine without local anaesthesic in patients undergoing major surgery: meta-analysis of randomized trials. Br JAnaesth. 2009; 102(2):156-167.

14. Borendal Wodlin N, Nilsson L, Kjølhede P; GASPI Study Group. The impact of mode of anaesthesia on postoperative recovery from fast-track abdominal hysterectomy: a randomised clinical trial. BJOG. 2011;118(3): 299-308.

15. El Sherif FA, Othman AH, Abd El-Rahman AM, Taha O. Effect of adding intrathecal morphine to a multimodal analgesic regimen for postoperative pain management after laparoscopic bariatric surgery: a prospective, double-blind, randomized controlled trial. Br J Pain. 2016; 10(4):209-216.

16. Ummenhofer WC, Arends RH, Shen DD, Bernards CM. Comparative spinal distribution and clearance kinetics of intrathecally administered morphine, fentanyl, alfentanil, and sufentanil. Anesthesiology. 2000; 92(3):739-753. 
17. Bujedo BM, Santos SG, Azpiazu AU. A review of epidural and intrathecal opioids used in the management of postoperative pain. J Opioid Manag. 2012;8(3):177-192.

18. Rathmell JP, Pino CA, Taylor R, Patrin T, Viani BA. Intrathecal morphine for postoperative analgesia: a randomized, controlled, dose-ranging study after hip and knee arthroplasty. Anesth Analg. 2003;97(5):1452-1457.

19. Fléron MH, Weiskopf RB, Bertrand M, et al. A comparison of intrathecal opioid and intravenous analgesia for the incidence of cardiovascular, respiratory, and renal complications after abdominal aortic surgery. Anesth Analg. 2003;97(1):2-12.

20. Kehlet H, Wilkinson RC, Fischer HB, Camu F; Prospect Working Group. PROSPECT: evidence-based, procedure-specific postoperative pain management. Best Pract Res Clin Anaesthesiol. 2007;21(1):149-159.

21. Koppert W. Opioid-induced hyperalgesia - pathophysiology and clinical relevance. Acute Pain. 2007;9:21-34.

22. Chu LF, Angst MS, Clark D. Opioid-induced hyperalgesia in humans: molecular mechanisms and clinical considerations. Clin J Pain. 2008;24(6):479-496.

23. Parisod E, Siddall PJ, Viney M, McClelland JM, Cousins MJ. Allodynia after acute intrathecal morphine administration in a patient with neuropathic pain after spinal cord injury. Anesth Analg. 2003;97:183-186.

24. Laulin JP, Larcher A, Célèrier E, Le Moal M, Simonnet G. Long-lasting increased pain sensitivity in rat following exposure to heroin for the first time. Eur J Neurosci. 1998;10:782-785.

25. Brennum J, Dahl JB, Møiniche S, Arendt-Nielsen L. Quantitative sensory examination of epidural anaesthesia and analgesia in man: effects of preand post-traumatic morphine on hyperalgesia. Pain. 1994;59(2):261-271.

26. Koppert W, Likar R, Geisslinger G, Zeck S, Schmelz M, Sittl R. Peripheral antihyperalgesic effect of morphine to heat, but not mechanical, stimulation in healthy volunteers after ultraviolet-B irradiation. Anesth Analg. 1999;88(1):117-122.

27. Fenten MG, Keijzer BA, Stienstra R. No clinically relevant advantage of intrathecal morphine in total hip arthroplasty? Acta Anaesthesiol Belg. 2014;65(4):161-166.

28. Olive DJ, Barrington MJ, Simone SA, Kluger R. A randomised controlled trial comparing three analgesia regimens following total knee joint replacement: continuous femoral nerve block, intrathecal morphine or both. Anaesth Intensive Care. 2015;43(4):454-460.

29. Essving P, Axelsson K, Åberg E, Spännar H, Gupta A, Lundin A. Local infiltration analgesia versus intrathecal morphine for postoperative pain management after total knee arthroplasty: a randomized controlled trial. Anesth Analg. 2011;113(4):926-933.
30. Lee JH, Park JH, Kil HK, Choi SH, Noh SH, Koo BN. Efficacy of intrathecal morphine combined with intravenous analgesia versus thoracic epidural analgesia after gastrectomy. Yonsei Med J. 2014;55(4): 1106-1114.

31. Lee SH, Gwak MS, Choi SJ, et al. Prospective, randomized study of ropivacaine wound infusion versus intrathecal morphine with intravenous fentanyl for analgesia in living donors for liver transplantation. Liver Transpl. 2013;19(9):1036-1045.

32. Moriyama K, Ohashi Y, Motoyasu A, Ando T, Moriyama K, Yorozu T. Intrathecal administration of morphine decreases persistent pain after cesarean section: a prospective observational study. PLoS One. 2016; 11(5):e0155114.

33. Fan C, Zhang D, Zhang CH. On sample size of the Kruskal-Wallis test with application to a mouse peritoneal cavity study. Biometrics. 2011; 67(1):213-224.

34. Beswick AD, Wylde V, Gooberman-Hill R, Blom A, Dieppe P. What proportion of patients report long-term pain after total hip or knee replacement for osteoarthritis? A systematic review of prospective studies in unselected patients. BMJ Open. 2012;2(1):e000435.

35. Nilsdotter AK, Petersson IF, Roos EM, Lohmander LS. Predictors of patient relevant outcome after total hip replacement for osteoarthritis: a prospective study. Ann Rheum Dis. 2003;62(10):923-930.

36. Jones CA, Beaupre LA, Johnston DW, Suarez-Almazor ME. Total joint arthroplasties: current concepts of patient outcomes after surgery. Rheum Dis Clin North Am. 2007;33(1):71-86.

37. Salinas FV, Liu SS, Mulroy MF. The effect of single-injection femoral nerve block versus continuous femoral nerve block after total knee arthroplasty of hospital length of stay and long-term functional recovery within an established clinical pathway. Anesth Analg. 2006;102: 1234-1239.

38. Benditz A, Greimel F, Auer P, et al. Can consistent benchmarking within a standardized pain management concept decrease postoperative pain after total hip arthroplasty? A prospective cohort study including 367 patients. J Pain Res. 2016;19(9):1205-1213.

39. Benditz A, Drescher J, Greimel F, et al. Implementing a benchmarking and feedback concept decreases postoperative pain after total knee arthroplasty: a prospective study including 256 patients. Sci Rep. 2016;6:38218

40. Liu SS, Buvanendran A, Rathmell JP, et al. A cross-sectional survey on prevalence and risk factors for persistent postsurgical pain 1 year after total hip and knee replacement. Reg Anesth Pain Med. 2012;37(4): $415-422$.

\section{Journal of Pain Research}

\section{Publish your work in this journal}

The Journal of Pain Research is an international, peer reviewed, open access, online journal that welcomes laboratory and clinical findings in the fields of pain research and the prevention and management of pain. Original research, reviews, symposium reports, hypothesis formation and commentaries are all considered for publication.

\section{Dovepress}

The manuscript management system is completely online and includes a very quick and fair peer-review system, which is all easy to use. Visit http://www.dovepress.com/testimonials.php to read real quotes from published authors. 